\title{
The imprint of crustal density heterogeneities on regional seismic wave propagation
}

\author{
Agnieszka Płonka $^{1}$, Nienke Blom ${ }^{1}$, and Andreas Fichtner ${ }^{2}$ \\ ${ }^{1}$ Department of Earth Sciences, Utrecht University, Utrecht, the Netherlands \\ ${ }^{2}$ Department of Earth Sciences, ETH Zurich, Zurich, Switzerland \\ Correspondence to: Agnieszka Plonka (a.i.plonka@uu.nl)
}

Received: 2 May 2016 - Published in Solid Earth Discuss.: 18 May 2016

Revised: 24 October 2016 - Accepted: 7 November 2016 - Published: 29 November 2016

\begin{abstract}
Density heterogeneities are the source of mass transport in the Earth. However, the 3-D density structure remains poorly constrained because travel times of seismic waves are only weakly sensitive to density. Inspired by recent developments in seismic waveform tomography, we investigate whether the visibility of 3-D density heterogeneities may be improved by inverting not only travel times of specific seismic phases but complete seismograms.

As a first step in this direction, we perform numerical experiments to estimate the effect of 3-D crustal density heterogeneities on regional seismic wave propagation. While a finite number of numerical experiments may not capture the full range of possible scenarios, our results still indicate that realistic crustal density variations may lead to travel-time shifts of up to $\sim 1 \mathrm{~s}$ and amplitude variations of several tens of percent over propagation distances of $\sim 1000 \mathrm{~km}$. Both amplitude and travel-time variations increase with increasing epicentral distance and increasing medium complexity, i.e. decreasing correlation length of the heterogeneities. They are practically negligible when the correlation length of the heterogeneities is much larger than the wavelength. However, when the correlation length approaches the wavelength, density-induced waveform perturbations become prominent. Recent regional-scale full-waveform inversions that resolve structure at the scale of a wavelength already reach this regime.

Our numerical experiments suggest that waveform perturbations induced by realistic crustal density variations can be observed in high-quality regional seismic data. While density-induced travel-time differences will often be small, amplitude variations exceeding $\pm 10 \%$ are comparable to those induced by 3-D velocity structure and attenuation.
\end{abstract}

While these results certainly encourage more research on the development of 3-D density tomography, they also suggest that current full-waveform inversions that use amplitude information may be biased due to the neglect of 3-D variations in density.

\section{Introduction}

Lateral variations in density are the driving force behind mass transport in the Earth, from crust to core (e.g. Kennett and Bunge, 2008; Turcotte and Schubert, 2014). They are the source of mantle convection, including the ascent of superplumes and the subduction of lithospheric plates. Knowledge of density is essential to discriminate between compositional and thermal heterogeneities (e.g. Trampert et al., 2004; Mosca et al., 2012), infer the nature of continental lithosphere (e.g. Jordan, 1975, 1978) or understand the relation between mantle convection and surface tectonics (e.g. Bunge et al., 2003; Liu and Gurnis, 2008; Warners-Ruckstuhl et al., 2012). Despite its outstanding importance for the solidEarth sciences, the 3-D density structure of our planet remains poorly constrained.

\subsection{The (in)sensitivity of seismic data to 3-D density variations}

Unlike seismic velocities that can be inferred from the travel times of elastic waves, unambiguous information on density is difficult to find in most seismic observables.

Within the framework of seismic ray theory (e.g. Cerveny, 2001), seismic travel times are particularly insensitive 
to density variations. In finite-frequency theory, the sensitivity of body waves to density is non-zero, but mostly confined to the immediate vicinity of sources and receivers (e.g. Tromp et al., 2005; Fichtner, 2010). The physical origin of this nearly complete absence of sensitivity lies in the scattering characteristics of density heterogeneities. When a body wave reaches a density perturbation, the resulting scattered wave propagates backwards, meaning that it cannot interfere with the incident wave unless the heterogeneity is located within one wavelength from either source or receiver (Wu and Aki, 1985; Tarantola, 1986; Trampert and Fichtner, 2013). This is in contrast to the scattered wave caused by a velocity heterogeneity, which propagates along with the incident wave, thereby leading to a finite-frequency travel-time shift (e.g. Tong et al., 1998; Marquering et al., 1999; Dahlen et al., 2000). Since scattered waves caused by density heterogeneities must exist, one may conclude that seismograms in general are sensitive to density variations, but this information cannot be contained in direct body wave travel times.

Unlike body wave travel times, the frequency-dependent travel times of Rayleigh waves reveal significant non-zero sensitivity to density variations (e.g. Takeuchi and Saito, 1972; Aki and Richards, 2002). The origin of this sensitivity can be understood intuitively with the mode-ray duality. Rayleigh waves can be seen as constructively interfering $P$ SV waves that reflect multiple times off the free surface. The reflection coefficient depends on density in the vicinity of the surface, thereby affecting the dispersion properties of the interference pattern. Unfortunately, Rayleigh wave sensitivity to density is strongly oscillatory, which leads to cancellation effects that leave little effective sensitivity to larger-scale variations.

At the long-period end of the seismic spectrum, the gravest normal modes of the Earth are sensitive to long-wavelength density structure as a result of the gravitational restoring force (Woodhouse, 1988; Dahlen and Tromp, 1998; Woodhouse and Deuss, 2007). This may be used to constrain density variations in the lower mantle where a low-degree structure is known to be dominant (e.g. Dziewoński et al., 1977; Becker and Boschi, 2002).

\subsection{Previous work and possible future directions}

Despite these difficulties, various attempts have been made to constrain 3-D density structure in the Earth. On the global scale, geodynamic data, including estimates of plate motion history and the location of subducting slabs, may be used to constrain the broad distribution of density in the mantle (Richards and Lithgow-Bertelloni, 1993; Nataf and Ricard, 1996; Simmons et al., 2010). Seismic constraints on 3 -D density variations in the lower mantle were first presented by Ishii and Tromp $(1999,2001,2004)$ based on longperiod normal-mode measurements. The robustness of their results has, however, been questioned by various authors (e.g. Resovsky and Ritzwoller, 1999; Romanowicz, 2001; Kuo and Romanowicz, 2002; Resovsky and Trampert, 2002). Recently, Koelemeijer et al. (2016) have shown that density estimates from previously used normal-mode data are not robust. However, with the incorporation of the latest data, these inferences can be improved significantly.

On regional scales, several authors jointly inverted bodywave travel times and gravity data under the assumption that seismic velocities and density are almost uniformly scaled to each other (e.g. Tondi et al., 2000, 2009; Maceira andAmmon, 2009). While correct for purely thermal density variations, this assumption prevents the detection of those interesting cases where velocities and density are not simply scaled due to the presence of compositional heterogeneities.

With the steadily increasing quality of seismic data, new observables with sensitivity to $3-\mathrm{D}$ density variations are becoming sufficiently robust. Lin et al. (2012); Lin and Tsai (2012) propose using Rayleigh-wave ellipticity and local amplification measurements to estimate lithospheric density variations. The design of seismic observables with maximum sensitivity to density and minimum trade-offs to other parameters, e.g. velocities, has been suggested by Bernauer et al. (2014).

In addition to improving data quality, new opportunities may arise from the development of full-waveform inversion techniques that are capable of exploiting complete seismograms without being restricted to the well-known seismic phases (e.g. Chen et al., 2007; Fichtner et al., 2009; Tape et al., 2010; Fichtner et al., 2013). As shown by Rickers et al. $(2012,2013)$, the exploitation of scattered waves in fullwaveform inversion can lead to substantial improvements in regional 3-D velocity images. However, the potential of fullwaveform inversion to better constrain density variations in the crust and upper mantle remains largely unexplored.

\subsection{Outline}

As a first step towards full-waveform inversion for regional density structure, we present a study on the imprint of 3-D density heterogeneities in the crust on seismic wave propagation in the period range from 8 to $50 \mathrm{~s}$. For this, we conduct a series of numerical experiments, where we analyse seismic wave propagation through random Earth models with variable complexity, i.e. correlation length scale. These models are designed to represent a range of plausible 3-D heterogeneous crustal environments. While wave propagation through random media has been widely used to quantify the effect of velocity heterogeneities (e.g. Frankel and Clayton, 1986; Frankel, 1989; Igel and Gudmundsson, 1997; Furumura and Kennett, 2005; Kennett and Furumura, 2008; Meschede and Romanowicz, 2015), variations in density have so far not been considered. Our experiments are intended to (i) provide rough estimates of the amplitude and travel-time variations related to realistic density variations, and (ii) better understand the physics behind density-induced waveform perturbations. 


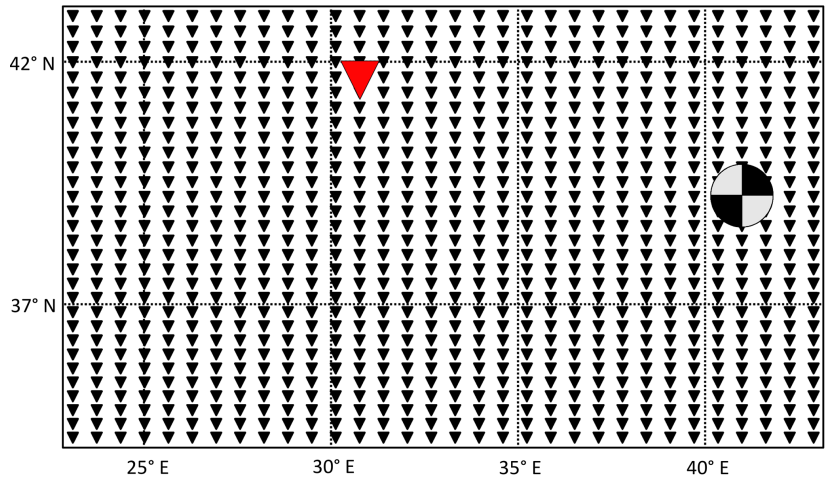

Figure 1. The grid of receivers (black triangles) on the surface of the computational domain. The source is located at $5 \mathrm{~km}$ depth; its location and orientation are indicated by the beach-ball plot. The receiver marked by a large red triangle and at an epicentral distance of $910 \mathrm{~km}$ is used for the examples presented in Sects. 3.1 and 3.2.

Following a presentation of the numerical setup, we will present detailed analyses of travel time and amplitude variations induced by 3-D crustal density heterogeneities. We expect scattering to be the dominant mechanism by which density heterogeneities influence the seismic signal. Scattering is most effective when scatterers are of similar size or smaller than the wavelength, which is why we will study the influence of frequency, propagation distance and medium complexity. Being focused on a future full-waveform inversion for density, we do not consider specific seismic phases, but try to provide ensemble estimates of waveform perturbations. Given the complexity of regional-scale seismic waveforms at periods below $\sim 20 \mathrm{~s}$, it is clear that this analysis can never be complete and exhaustive. It will, however, provide a first crude estimate of the impact of crustal density structure on seismic wave propagation.

\section{Setup of the numerical experiments}

\subsection{Numerical wave propagation}

To assess the impact of 3-D density heterogeneities in the crust on seismic wave propagation, we compute numerical solutions to the elastic wave equation (e.g. Kennett, 2001; Aki and Richards, 2002)

$\rho(\mathbf{x}) \partial_{t}^{2} u_{i}(\mathbf{x}, t)-\partial_{j}\left[c_{i j k l}(\mathbf{x}, t) \cdot \partial_{k} u_{l}(\mathbf{x}, t)\right]=f_{i}(\mathbf{x}, t)$,

which relates mass density $\rho$, the elastic tensor $c_{i j k l}$, and an external force $f_{i}$ to the displacement field $u_{i}$. With our focus being on regional wave propagation at periods below $50 \mathrm{~s}$, we can safely ignore the Earth's rotation and self-gravitation. Furthermore, we restrict ourselves to an isotropic rheology.

For the numerical solution of Eq. (1), we employ the spectral-element solver SES3-D (Fichtner et al., 2009; Gokhberg and Fichtner, 2016). The spectral-element method, widely used in seismological research, allows us to compute accurate numerical solutions in the presence of strong 3-D heterogeneities, without requiring special treatment of the free surface (e.g. Faccioli et al., 1996; Komatitsch and Vilotte, 1998; Komatitsch and Tromp, 1999; Peter et al., 2011; Cupillard et al., 2012).

Our computational domain is a spherical section that is $\sim 2000$ by $\sim 1000 \mathrm{~km}$ wide and $500 \mathrm{~km}$ deep. As a background model we use the radially symmetric preliminary reference Earth model (Dziewoński and Anderson, 1981), where we replace the original $24 \mathrm{~km}$ thick crust by a $40 \mathrm{~km}$ thick crust that better represents continental structure. Since the number of receivers has no significant impact on the computational costs of the numerical simulations, we use a dense grid of 930 receivers, distributed evenly across the surface of the computational domain. In the wave field simulations, we calculate $700 \mathrm{~s}$ long velocity seismograms from a strike-slip source. The complete setup is shown in Fig. 1.

\subsection{Random media generation}

Because the true 3-D density structure of the crust is insufficiently constrained, we use synthetic random density models in our numerical wave propagation experiments. For this, we superimpose random velocity and density variations with pre-defined correlation lengths in the horizontal and vertical directions onto the crustal part of the background model, i.e. the upper $40 \mathrm{~km}$. The spatial variations in velocity and density are statistically uncorrelated, meaning that the spatial correlation averaged over many realisations is negligibly small. The individual realisations considered in this work have non-zero correlation. In Appendix Sect. A, we summarise the computation of 3-D random models based on the widely used Fourier method.

To ensure that the amplitudes of velocity and density variations are realistic, we combine information from tomographic models and empirical velocity-density scalings. For this, we compute the root-mean square (rms) of the $S$ velocity variations in the regional crustal model of Anatolia obtained by Fichtner et al. (2013), using full-waveform inversion. $S$ velocities at crustal depths in this model are resolved on length scales of $\sim 25 \mathrm{~km}$. Using the empirical scaling relations between crustal velocities and density of Brocher (2005), we then obtain suitable ranges for variations in $P$ velocity and density. The resulting rms variations are $260 \mathrm{~m} \mathrm{~s}^{-1}$ for $S$ velocity, $460 \mathrm{~m} \mathrm{~s}^{-1}$ for $P$ velocity, and $80 \mathrm{~kg} \mathrm{~m}^{-3}$ for density. Two particular realisations of random density variations with different correlation lengths are shown in Fig. 2. While the random models are intended to represent plausible variations of crustal structure, there exists of course uncertainty related to the poorly known amplitude spectrum of these variations in the real Earth, and the range of different velocity-density scalings proposed in the literature. We discuss these issues in more detail in Sect. 4.2. We note that random velocity and density models used in our simulations 

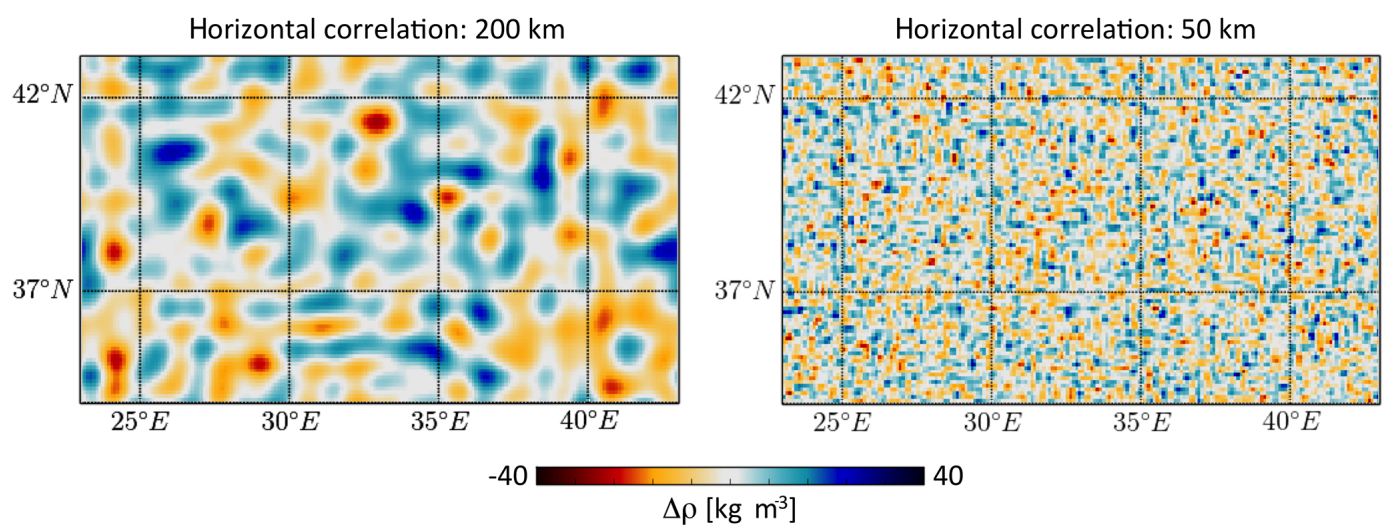

Figure 2. Realisations of random density variations. Left: $200 \mathrm{~km}$ lateral correlation length, $20 \mathrm{~km}$ vertical correlation length. Right: $50 \mathrm{~km}$ lateral correlation length, $10 \mathrm{~km}$ vertical correlation length.

are on purpose spatially uncorrelated. Empirical velocitydensity scalings are used only to determine plausible rms variations.

\subsection{Quantification of waveform differences}

In our numerical experiments, we use media with homogeneous crustal density and random 3-D variations in $P$ and $S$ velocities as reference. We then compare synthetic seismograms from the reference medium with synthetic seismograms from a medium where random variations in density are added.

Since our ultimate goal is to use complete threecomponent seismograms to constrain density in the Earth, we do not compare isolated and well-defined seismic phases. Instead, we compute time- and frequency-dependent traveltime and amplitude differences. For this, we bandpass-filter the seismograms into a pre-defined frequency band and apply a zero-centred moving window $w(t)$ that transforms a component of a seismogram $u(t)$ into its windowed version $\hat{u}_{\tau}(t)=w(t-\tau) u(t)$. The travel-time difference $\delta T$ as a function of $\tau$ is then defined as the argument of the maximum of the cross-correlation:

$\delta T(\tau)=\underset{t}{\operatorname{argmax}} \int \hat{u}_{\tau}^{\mathrm{ref}}\left(t^{\prime}\right) \hat{u}_{\tau}\left(t+t^{\prime}\right) \mathrm{d} t^{\prime}$,

where $\hat{u}_{\tau}^{\text {ref }}$ denotes the windowed seismogram for the reference medium with homogeneous crustal density. In the case of $\delta T<0$, the wave for 3-D heterogeneous density arrives earlier than the reference wave, and vice versa. Similarly, we measure relative amplitude variations $\delta A$ as a function of time:

$\delta A(\tau)=\frac{\sqrt{\int\left[\hat{u}_{\tau}(t)\right]^{2} \mathrm{~d} t}-\sqrt{\int\left[\hat{u}_{\tau}^{\mathrm{ref}}(t)\right]^{2} \mathrm{~d} t}}{\sqrt{\int\left[\hat{u}_{\tau}^{\mathrm{ref}}(t)\right]^{2} \mathrm{~d} t}}$.

In the following sections, we consider three frequency bands of variable width: $0.02-0.125 \mathrm{~Hz}(8-50 \mathrm{~s}), 0.02-0.067 \mathrm{~Hz}$
Table 1. Lateral and vertical correlation lengths of random medium variations used to assess the influence of medium complexity.

\begin{tabular}{lr}
\hline $\begin{array}{l}\text { Lateral correlation } \\
\text { length }[\mathrm{km}]\end{array}$ & $\begin{array}{r}\text { Vertical correlation } \\
\text { length }[\mathrm{km}]\end{array}$ \\
\hline 1000 & 100 \\
200 & 20 \\
50 & 10 \\
\hline
\end{tabular}

$(15-50 \mathrm{~s})$, and $0.02-0.04 \mathrm{~Hz}(25-50 \mathrm{~s})$. The $w(t)$ Gaussian time windows corresponding to those frequency bands have standard deviations of 8,15 and $25 \mathrm{~s}$, respectively. To stabilise the measurements, we exclude those parts of the synthetic seismograms where the average amplitude within a time window is below $5 \%$ of the maximum within the complete trace.

While more information-rich quantifications of seismic waveform differences may be constructed, for instance on the basis of wavelet transforms (e.g. Kristekova et al., 2006, 2009), we prefer the travel time and amplitude differences defined in Eqs. (2) and (3) for their robustness and ease of interpretation. Similar quantifiers of waveform differences are frequently used in full-waveform inversion (e.g. Fichtner et al., 2008; van Leeuwen and Mulder, 2010; Bozdağ et al., 2011; Rickers et al., 2012, 2013). Since density impacts $P$ and $S$ energies in the same way, there is no need for any component rotation.

\section{Impact of density heterogeneities on wave propagation}

In the following sections, we present a phenomenological study on the impact of crustal density heterogeneities for media with different horizontal and vertical correlation lengths, summarised in Table 1. For each experiment, we compare three-component seismograms in three different 
Bandpass: $0.020-0.125 \mathrm{~Hz}$

- Homogeneous crustal density (reference) - Heterogeneous crustal density
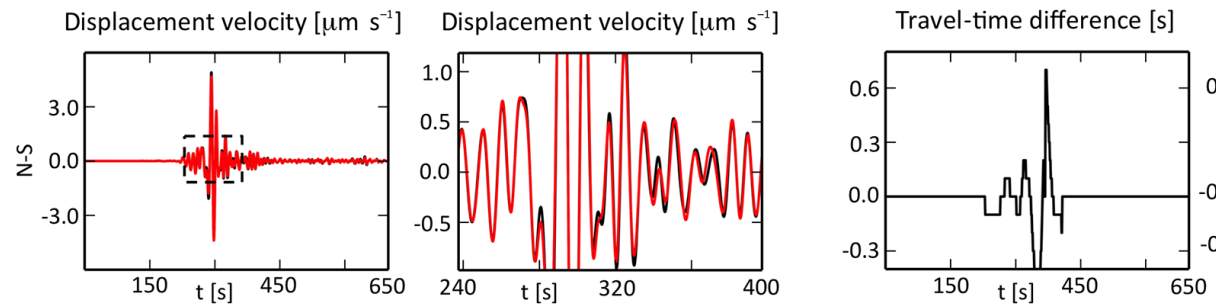

Rel. amplitude difference [1]
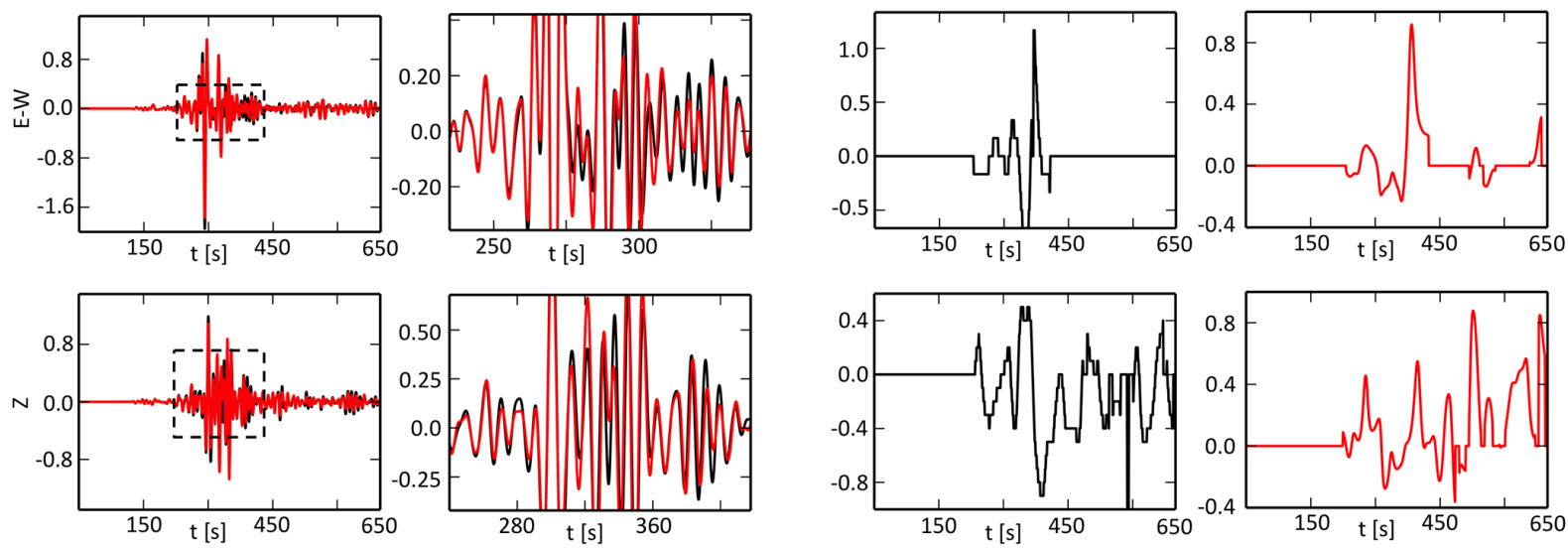

Figure 3. Comparison of synthetic seismograms for homogeneous and heterogeneous crustal densities in the broadest frequency band from 0.020 to $0.125 \mathrm{~Hz}(8-50 \mathrm{~s})$. The receiver is located at an intermediate epicentral distance of $910 \mathrm{~km}$, and is marked by a red triangle in Fig. 1 . The correlation lengths of the heterogeneities in velocities and density are $200 \mathrm{~km}$ in the horizontal and $20 \mathrm{~km}$ in the vertical directions. The first column displays the seismograms on the N-S, E-W and Z components for the reference medium with homogeneous crustal density (red) and a medium with random heterogeneous crustal density (black). For better visibility, a zoom into the interval indicated by the black-dashed box is shown in the second column. The third and fourth columns display the time-dependent travel time and relative amplitude difference of the two sets of seismograms shown to the left. Extreme travel-time differences due to cycle skips are cropped to enhance visibility of physically meaningful travel-time variations.

frequency bands: seismograms computed for a medium with 3-D random density variations are compared with seismograms for the reference medium with homogeneous crustal density. We specifically analyse the effects of frequency (Sect. 3.2), epicentral distance (Sect. 3.3), and medium complexity (Sect. 3.4). Here and in the following sections, we use "increasing complexity" as synonymous to "decreasing correlation length".

\subsection{A single-receiver example}

We start with the analysis of media with 200 lateral and $20 \mathrm{~km}$ vertical correlation length. This will serve as a baseline for later simulations with models that have either more or less complexity. Before attempting a more comprehensive analysis in the following sections, we consider a single receiver located at $910 \mathrm{~km}$ epicentral distance, marked by the red triangle in Fig. 1. Figure 3 shows a comparison of three-component seismograms for homogeneous and heterogeneous crustal densities in the broadest frequency band from 0.020 to $0.125 \mathrm{~Hz}(8-50 \mathrm{~s})$.
Waveform differences mostly tend to increase with increasing travel time, in accord with the expectation that (multiply) scattered waves should arrive later than the primary waves by which they have been excited. The magnitude of the time shifts are approximately independent of the component, reaching around $0.5 \mathrm{~s}$. Relative amplitude differences are largest on the $\mathrm{E}-\mathrm{W}$ and vertical components, where the displacement velocity itself is smallest. Therefore, lowamplitude scattered waves have the largest influence on the total amplitude. They regularly exceed $50 \%$ in both directions, meaning that amplitudes for the heterogeneous density crust can be either twice or half as large as for the medium with homogeneous crustal density. On the N-S component, where the displacement velocity is largest, relative amplitude differences vary between $\pm 10 \%$.

Figure 4 displays time shifts and relative amplitude differences for five different realisations of random media, at the same receiver and for a lower frequency band from 0.02 to $0.04 \mathrm{~Hz}$. Both travel time and amplitude variations differ significantly for different media, which has two important implications: (i) different media may be distinguished from each 
Dependence of waveform differences on random medium realisations
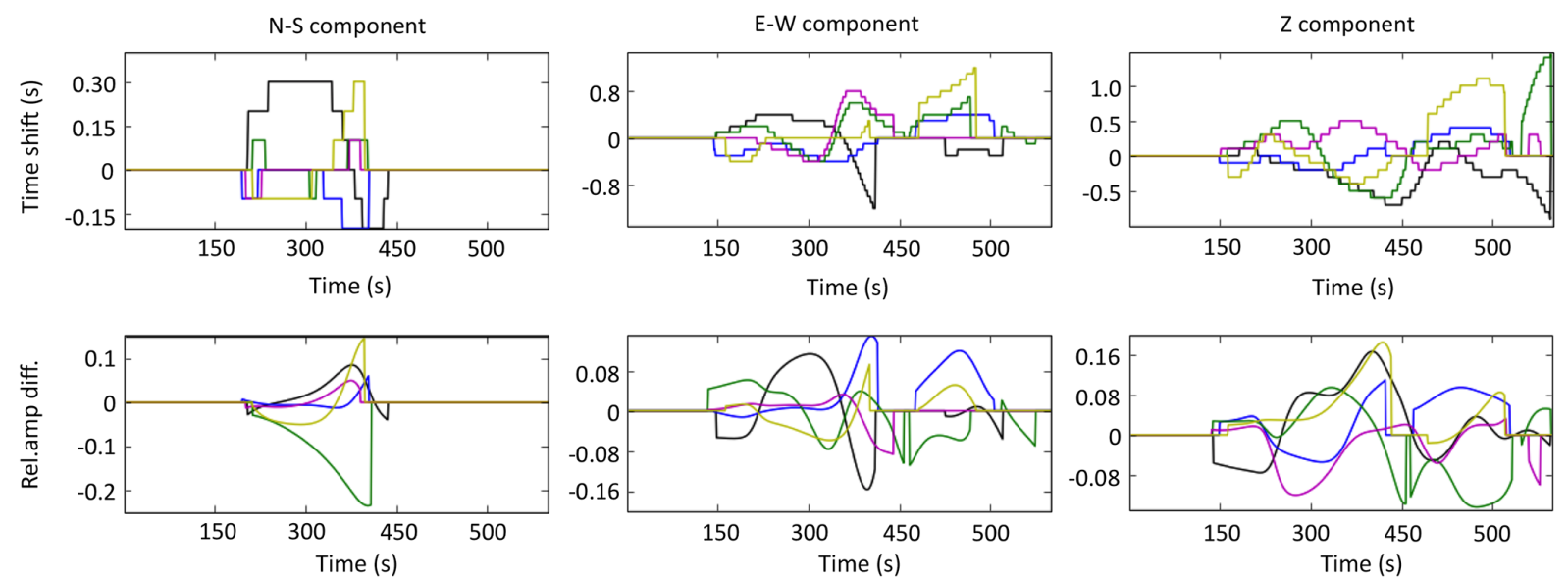

Figure 4. Component-wise time shifts and relative amplitude differences in the frequency band from 0.02 to $0.04 \mathrm{~Hz}$ for five random media realisations.

other, at least to some extent that remains to be quantified. (ii) To obtain statistically significant results in the present study, we must average travel time and amplitude variations over various random realisations. Based on our experience, five realisations are sufficient to obtain reliable results.

In addition to the dependence on the random velocity and density structure, Fig. 4 also reveals that the waveform differences in the lower frequency band from 0.02 to $0.04 \mathrm{~Hz}$ are on average smaller than for the higher frequency band from 0.020 to $0.125 \mathrm{~Hz}$. In the following section, we will investigate this frequency dependence in more detail.

\subsection{The effect of frequency}

A variant of Fig. 3 for the narrower and lower frequency band from 0.02 to $0.04 \mathrm{~Hz}(25-50 \mathrm{~s})$ is shown in Fig. 5. While relative amplitude differences are markedly smaller than at higher frequencies, the travel-time differences are still comparable.

This preliminary, and mostly visual, analysis of frequency dependence indicates that waveform differences are primarily caused by scattering that transfers energy from the largeamplitude $\mathrm{N}-\mathrm{S}$ component onto the smaller-amplitude $\mathrm{E}-\mathrm{W}$ and vertical components. Constructive and destructive interference between primary and scattered waves may cause the wave amplitudes to deviate in both directions. An increase of amplitudes may be further supported by additional wave focusing induced by 3-D density heterogeneities. The approximate frequency independence of travel-time differences, however, can hardly be explained with basic wave propagation intuition.

To make our analysis more comprehensive and efficient, we compute histograms of time shifts and relative amplitude differences for all 930 stations in the receiver grid. In line with our future goal, which is to use full-waveform inver- sion to constrain 3-D density variations, we do not consider specific seismic phases, but longer time series that comprise body, surface and scattered waves. After calculating the misfits for all of the receivers of the grid, we stack their values into histograms, each histogram corresponding to a different frequency band. The values that we consider in the stacking procedure are measured up to $300 \mathrm{~s}$ after the first difference between the two waveforms (shortly after the first arrival of the $P$ wave). This ensures, firstly, that the difference in epicentral distance between receivers does not affect the histogram shape, and secondly, that most of the waveforms with large enough amplitudes are included, while excluding low-amplitude parts of the seismograms where numerical errors have a larger impact. In order to obtain representative results, we average the measurements of time shift and relative amplitude differences over five random media realisations. Histograms showing the effect of bandwidth on time shifts and relative amplitude differences are displayed in Fig. 6.

Relative amplitude differences for the broader frequency band, i.e. for frequencies that are on average higher, have a considerably larger spread than at lower frequencies. In the $0.02-0.125 \mathrm{~Hz}$ band, the standard deviation of the amplitude differences is 0.15 . Amplitudes can be both smaller and larger than in the reference scenario with homogeneous crustal density. Within the lower frequency band from 0.02 to $0.04 \mathrm{~Hz}$, the standard deviation of the amplitude differences is reduced to 0.07 , suggesting that the single-station analysis from Figs. 3 and 5 has more general validity.

The dependence of time shifts on frequency is more complex, as shown in the left panel of Fig. 6 and in Table 2. The standard deviation reaches $0.2 \mathrm{~s}$ for the two highest frequency bands $(0.02-0.125$ and $0.02-0.067 \mathrm{~Hz})$, and surprisingly increases to $0.38 \mathrm{~s}$ for the lowermost frequency band from 0.02 to $0.04 \mathrm{~Hz}$. 


\section{Homogeneous crustal density (reference) - Heterogeneous crustal density}

Displacement velocity $\left[u m \mathrm{~s}^{-1}\right] \quad$ Displacement velocity $\left[u \mathrm{~m} \mathrm{~s}^{-1}\right]$
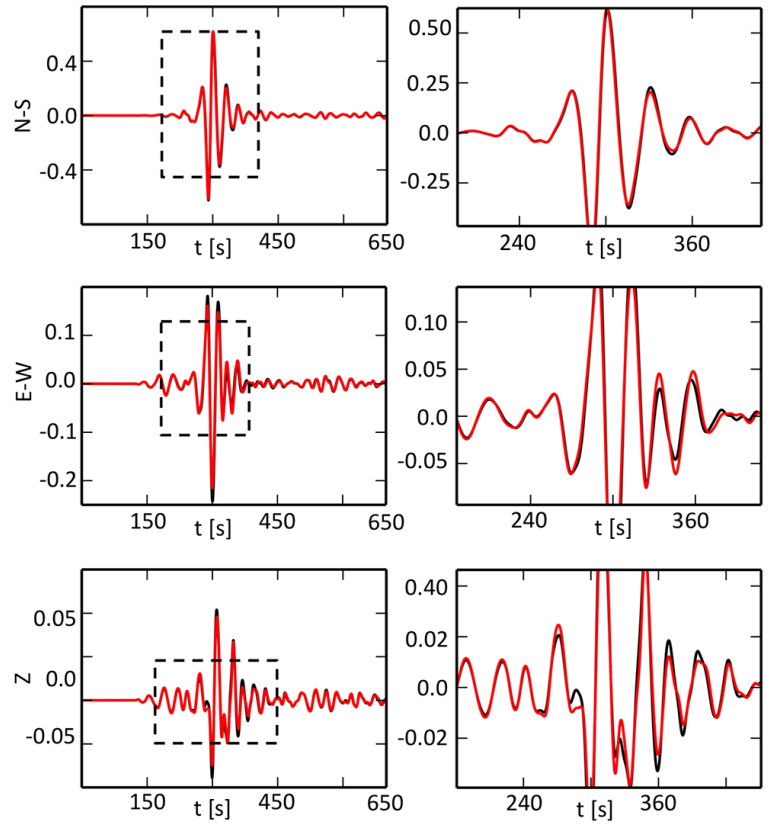

Travel-time difference [s]

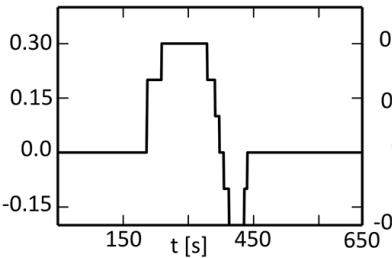

Rel. amplitude difference [1]
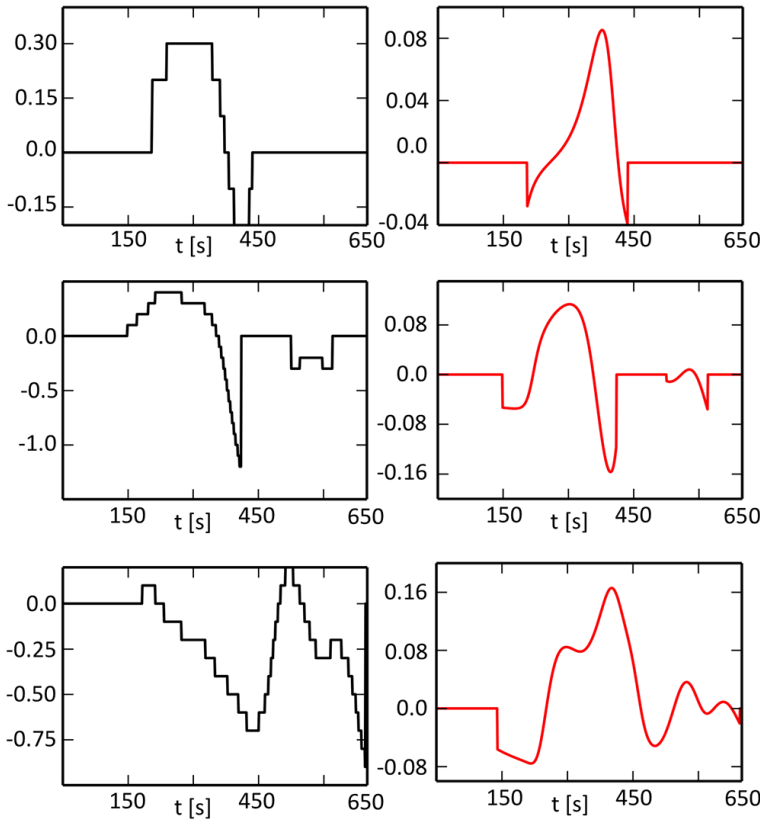

Figure 5. The same as in Fig. 3 but for the narrowest frequency band from 0.02 to $0.04 \mathrm{~Hz}(25-50 \mathrm{~s})$.
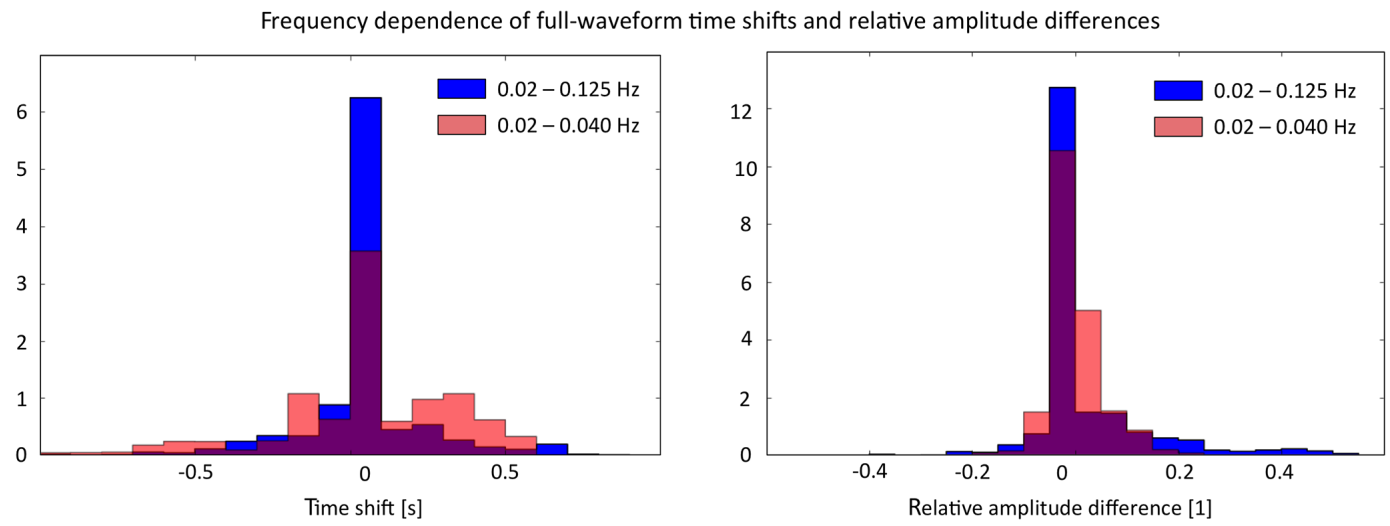

Figure 6. Normalised histograms of time shifts (left) and relative amplitude differences (right) averaged over five random media realisations with $200 \mathrm{~km}$ lateral and $20 \mathrm{~km}$ vertical correlation length. The lower frequency band from 0.02 to $0.04 \mathrm{~Hz}$ is shown in magenta, and the higher frequency and from 0.02 to $0.125 \mathrm{~Hz}$ in blue. Relative amplitude differences at higher frequencies (blue) have a visibly larger spread than at lower frequencies (magenta). The spreads of the time shift histograms are, however, comparable for both frequency bands. A summary of standard deviations in the histograms is provided in Table 2.

To investigate this phenomenon further, we show histograms for the lowest and highest frequency bands for a single random medium realisation and for the three different components in the top row of Fig. 7. We observe a distinct tail of reduced time shifts of $\sim 1 \mathrm{~s}$ on the E-W component and for the lowest frequency band. A similar observation can be made for two out of five random media, suggesting that these time shifts are not highly unlikely to be artefacts of an unusual random medium realisation. The bottom row of Fig. 7 shows a pair of synthetic seismograms at an epicentral distance of $\sim 1300 \mathrm{~km}$ that contributes to this tail of negative time shifts in the mentioned histograms - we show directly by plotting this exemplary pair that in the lower frequency band (from 0.02 to $0.04 \mathrm{~Hz}$ ), the waveforms for the 3-D heterogeneous crust arrive early by $\sim 1 \mathrm{~s}$. The tail of negative time shifts appears only for stations of distance between 1000 
Frequency-dependent full-waveform time shifts for a single random medium realisation
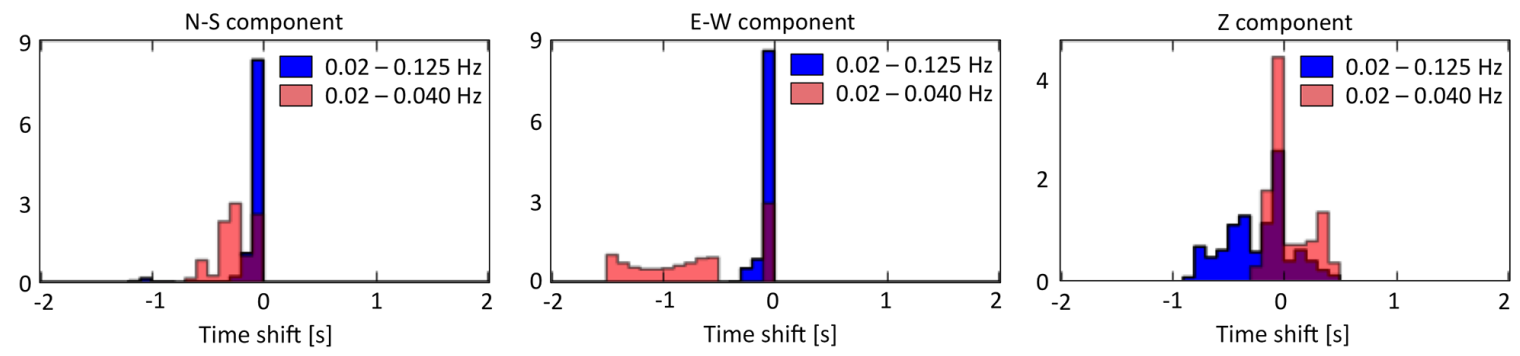

E-W component velocity seismograms
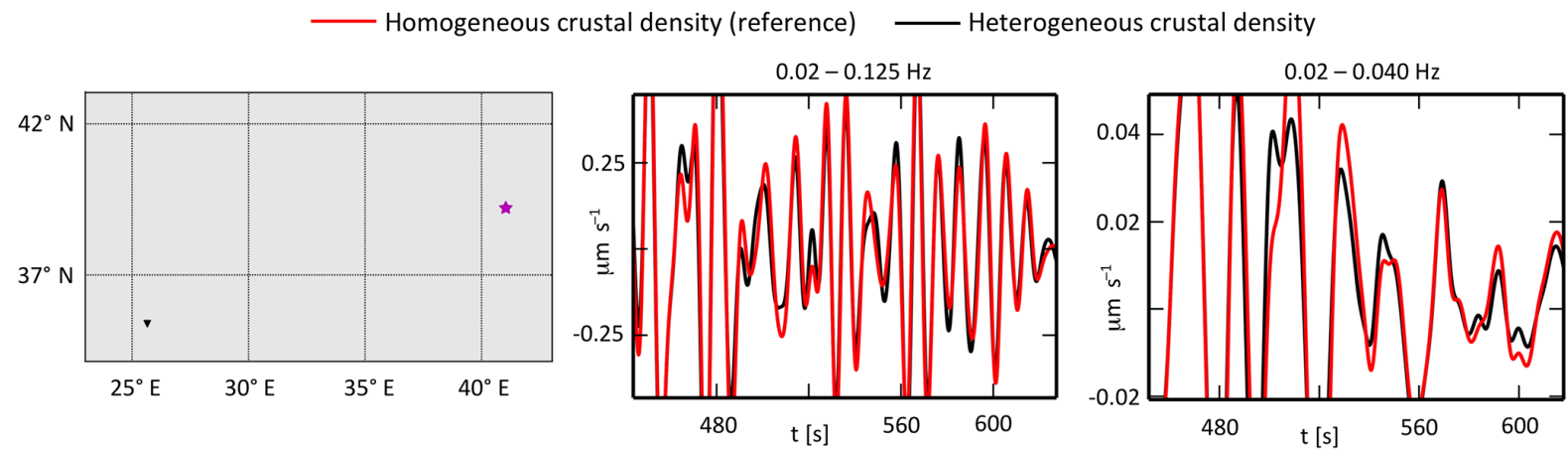

Figure 7. Top row: Normalised histograms of frequency-dependent time shifts for a single random medium realisation with 200 lateral and $20 \mathrm{~km}$ vertical correlation length. The lower frequency band from 0.02 to $0.04 \mathrm{~Hz}$ is shown in magenta, and the higher frequency and from 0.02 to $0.125 \mathrm{~Hz}$ in blue. The three different components are plotted separately. The isolated tail of reduced time shifts for the lowest frequency band on the E-W component was observed for two out of five random medium realisations, the average of which is shown in Fig. 6. Bottom row: Comparison of E-W component synthetic seismograms for the homogeneous reference crust (red) and the 3-D heterogeneous crust (black) for the source-receiver geometry shown in the left panel. In the lower frequency band from 0.02 to $0.04 \mathrm{~Hz}$ waves for the 3 -D heterogeneous crust arrive early by $\sim 1 \mathrm{~s}$, thus contributing to the negative time shift tail in the E-W component histogram shown above. For the higher frequency band from 0.02 to $0.125 \mathrm{~Hz}$, a similar effect is not visible.

and $1200 \mathrm{~km}$ (the far away stations) and is absent for stations of epicentral distance between 100 and $300 \mathrm{~km}$ (the stations close to the source). We examined this distance dependence further, using synthetic data from one of the numerical experiments contributing to the tail. For all the frequency bands for far away stations, the mean time shift value is shifted to negative values between -0.08 and $-0.14 \mathrm{~s}$. While the mean time-shift values are similar for all the frequencies in question, the standard deviation of time shift for the lowest frequency band is three times as big as for the other bands and reaches $0.8 \mathrm{~s}$. We do not observe any of those relations for the stations close to the source. A visual waveform comparison suggests that the travel-time differences may be a finitefrequency effect, meaning that waveform (amplitude) differences within short time intervals translate into time shifts when these are measured by cross-correlation within a finite frequency band. We discuss this aspect in more detail in Sect. 4.1.

\subsection{The effect of epicentral distance}

To investigate whether density-related travel time and amplitude differences are only local effects or accumulate with propagation distance, we plot histograms for stations in two different epicentral distance ranges: 100-300 and 1000$1200 \mathrm{~km}$. We again average over five random media realisations with 200 lateral and $20 \mathrm{~km}$ vertical correlation length. The results for the broadest frequency band from 0.02 to $0.125 \mathrm{~Hz}$ are shown in Fig. 8 .

For epicentral distances between 100 and $300 \mathrm{~km}$, the variance of the time shifts is $0.15 \mathrm{~s}$, and it increases to $0.64 \mathrm{~s}$ for epicentral distances between 1000 and $1200 \mathrm{~km}$. Similarly, the variance of relative amplitude differences increases from 0.007 to 0.111 . This indicates that waveform differences due to crustal density heterogeneities indeed accumulate with increasing epicentral distance, which is an essential prerequisite for the use of tomographic methods. 


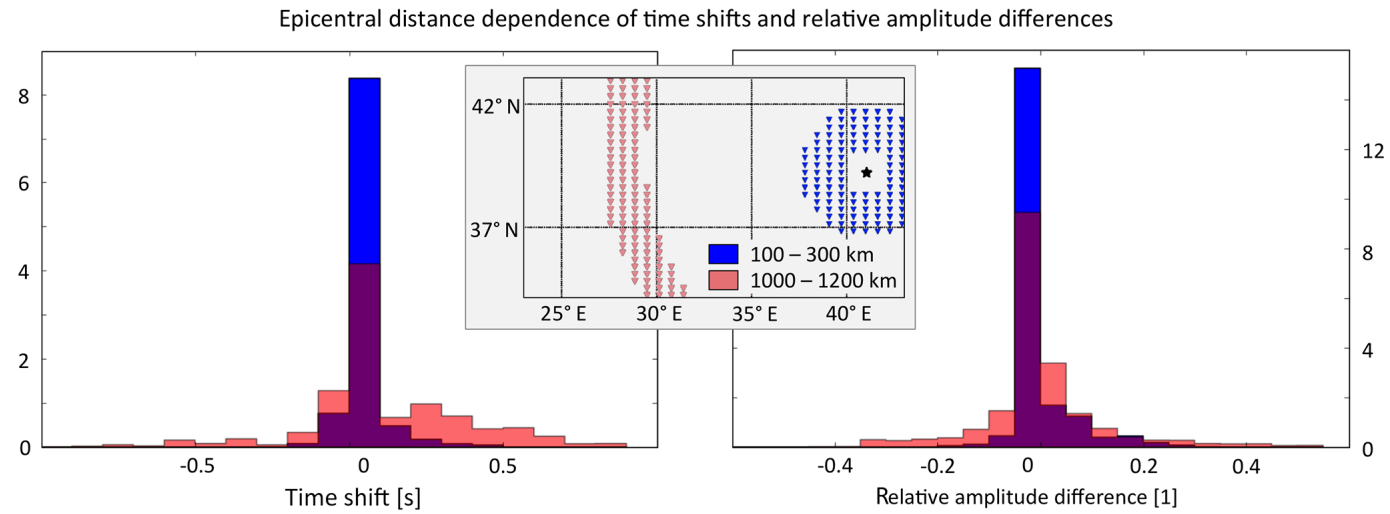

Figure 8. Normalised time shifts (left) and relative amplitude differences (right) for stations in two epicentral distance ranges: 100-300 (blue) and $1000-1200 \mathrm{~km}$ (magenta). The source-receiver configuration is shown in the inset. The frequency range is $0.02-0.125 \mathrm{~Hz}$. The spread of both time shifts and relative amplitude differences increases with epicentral distance, indicating that the observed waveform differences accumulate with increasing propagation distance instead of being a purely local effect.

Table 2. Standard deviations of time shifts and relative amplitude differences as a function of frequency bandwidth.

\begin{tabular}{lrr}
\hline Frequency band & $\begin{array}{r}\text { Standard deviation } \\
\text { of time shifts }\end{array}$ & $\begin{array}{r}\text { Standard deviation of } \\
\text { relative amplitude } \\
\text { differences }\end{array}$ \\
\hline $0.02-0.04 \mathrm{~Hz}$ & $0.38 \mathrm{~s}$ & 0.07 \\
$0.02-0.067 \mathrm{~Hz}$ & $0.20 \mathrm{~s}$ & 0.06 \\
$0.02-0.125 \mathrm{~Hz}$ & $0.19 \mathrm{~s}$ & 0.15 \\
\hline
\end{tabular}

\subsection{The effect of medium complexity}

In order to reveal the physical origin of the waveform differences, we consider random media with different lateral correlation lengths, listed in Table 1 . We again work with the broadest frequency band from 0.02 to $0.125 \mathrm{~Hz}$.

As shown in the top row of Fig. 9, the medium with 50 $\mathrm{km}$ lateral correlation length leads to a broad distribution of time shifts and relative amplitude differences, compared to the medium with $200 \mathrm{~km}$ lateral correlation used in the previous sections. The standard deviations of time shifts and relative amplitude differences reach values of $0.94 \mathrm{~s}$ and 0.20 , respectively. In contrast, travel time and amplitude variations for the smooth medium, shown in the bottom row of Fig. 9, are nearly zero for all times and for all receivers. Their standard deviations are $0.01 \mathrm{~s}$ and 0.01 , respectively.

The histograms in Fig. 9 indicate that waveform differences induced by 3 -D density variations occur mostly due to scattering which is most effective when heterogeneities are equal or smaller in size than the wavelength. In a medium with $50 \mathrm{~km}$ lateral correlation length, the size of heterogeneities is comparable to the wavelength of waves with a maximum frequency of $0.125 \mathrm{~Hz}$, and scattering becomes the dominant mechanism to perturb the wave field. In the smooth medium with $1000 \mathrm{~km}$ lateral correlation length, the domi- nant mechanism is transmission, which clearly has no significant impact on either travel times or amplitudes.

\section{Discussion}

\subsection{Seismic signatures of crustal density heterogeneities}

Our numerical experiments show that 3-D crustal density heterogeneities may lead to both positive and negative variations in the travel times and amplitudes of seismic waves. This indicates that 3-D density structure leaves an imprint on regional seismic wave fields that goes beyond simple scattering attenuation of the main arrivals.

To understand the effects which play a major role in wave propagation, we look first at the misfit histograms for different frequency bands (Sect. 3.2). Intuitively, the histogram for the frequency band in which more misfits are accumulated should have smaller zero peak and bigger spread. We would expect to observe more misfits for higher frequencies due to illumination of finer structures. However, as can be seen in Fig. 6, while the peak around zero is smaller for the lower frequency band for both time shifts and amplitude differences, the spread is comparable for time shifts and larger for the higher frequency band for amplitude differences. This may mean that we observe a variety of effects that affect wave propagation and have different impact on the observed seismograms for different frequency bands. Below we discuss this seemingly counterintuitive result.

Our lowest bandwidth has a peak frequency of $0.03 \mathrm{~Hz}$, which translates into a dominant wavelength of roughly $100 \mathrm{~km}$ for a wave travelling with the velocity of $3.2 \mathrm{~km} \mathrm{~s}^{-1}$ (the PREM value for crustal $S$ velocity). Since the lateral cross-correlation length of the random medium used in our setup is $200 \mathrm{~km}$, the lateral mean size of a scatterer should 

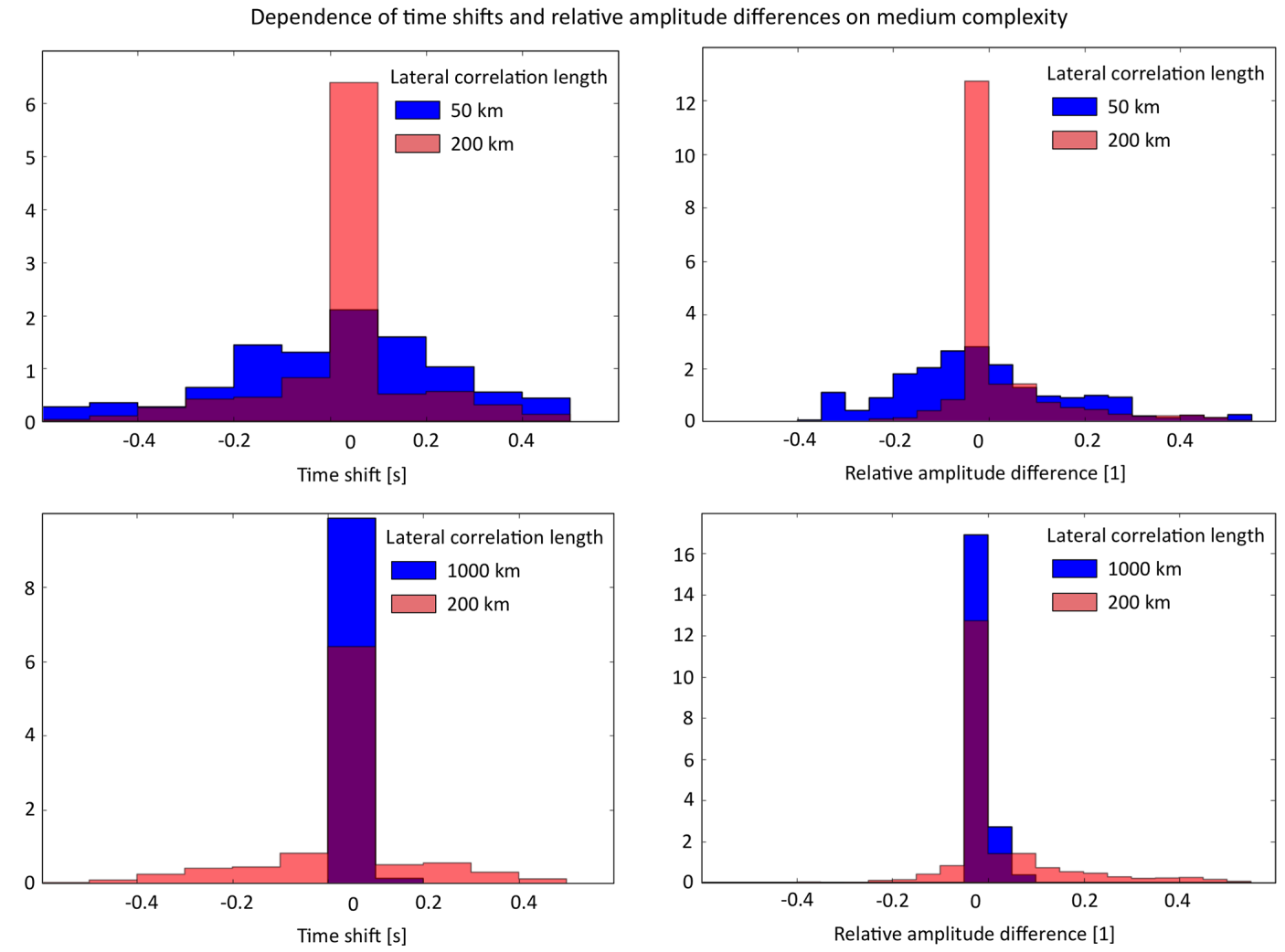

Figure 9. Histograms of time shifts (left) and relative amplitude differences (right) for a complex medium with $50 \mathrm{~km}$ lateral correlation length (top, blue) and a smooth medium with $1000 \mathrm{~km}$ lateral correlation length (bottom, blue). The medium with $200 \mathrm{~km}$ lateral correlation length, used in the previous sections, is used as reference and plotted in magenta. The frequency band is $0.02-0.125 \mathrm{~Hz}$. Both time shifts and relative amplitude differences grow considerably with growing complexity of the medium.

reach the value of around $100 \mathrm{~km}$, which matches the dominant wavelength of the lowest bandwidth. This would mean that in this bandwidth, we will observe resonant scattering for waves travelling horizontally with the velocity of around $3.2 \mathrm{~km} \mathrm{~s}^{-1}$. In consequence, due to more scattering, we will measure higher values of density-related misfits. Increasing the peak frequency to $0.07 \mathrm{~Hz}$, which is the peak frequency of our highest bandwidth $(0.02-0.125 \mathrm{~Hz})$, would decrease the dominant wavelength for an $S$ wave to around $50 \mathrm{~km}$, and thus move us away from resonance. In that case, with increasing frequency, scattering off heterogeneities becomes less significant in favour of transmission effects. That essentially means approaching the range of the infinite-bandwidth approximation of ray theory validity, in which we completely lose sensitivity to density. Smaller scattering in higher frequencies may mean that we will observe more misfits of values around zero, causing the histograms for the higher frequency band to have larger zero peaks.

An increase of the peak frequency of the bandwidth in our particular case means observing less scattering for certain waves, however, it also means a proportional increase in relative propagation distance. This implies that we will observe waveform differences accumulated for bigger number of wavelengths, and the amount of large non-zero densityrelated misfits will increase. This is an effect that changes the histogram shape in a way opposite to moving away from resonance. The larger propagation distance could then be one of the reasons behind the broader histogram spread for the higher frequency band.

Increasing the relative propagation distance is equivalent to moving further from the source, which is consistent with Sect. 3.3, where we show that density-related misfits accumulate with distance. The observation of more waveform differences with larger epicentral distance also suggests that the impact of density structure is not merely a local effect, but rather an integral over the complete wave path - an essential prerequisite for performing tomography.

While the results for different frequency bands are physically governed by the amount of scattering and the length of the relative propagation distance, and the results for different epicentral distances by the length of the propagation distance only, for various medium complexities we observe how important scattering is for sensitivity to density. The noticeable change in histogram shape in Fig. 9 is caused by much bigger amount of scattering for more complex media. The nearly complete absence of waveform differences 
Wavefield snapshots

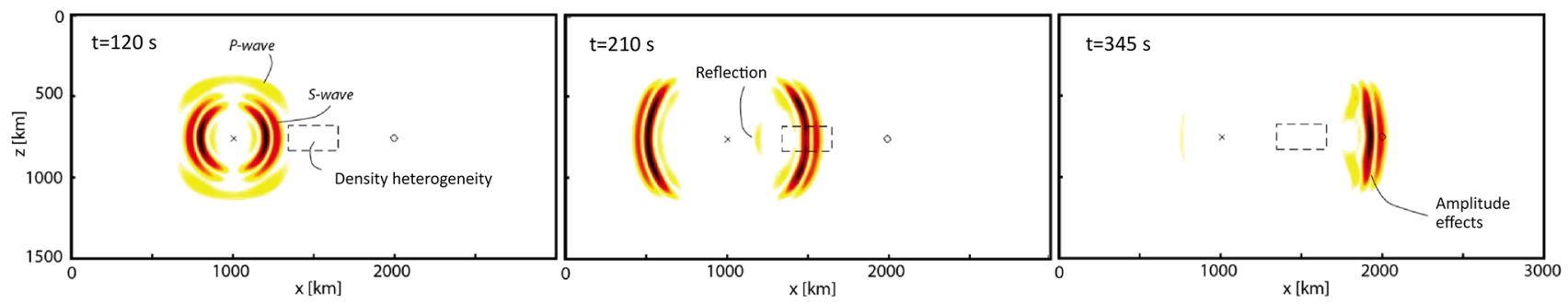

Waveforms without / with density heterogeneity $\left[10^{-4} \mathrm{~m} \mathrm{~s}^{-1}\right]$

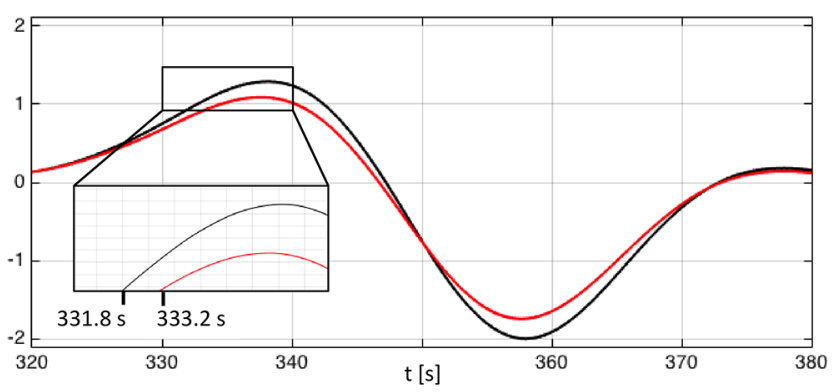

Waveform difference (with minus without density heterogeneity) $\left[10^{-5} \mathrm{~m} \mathrm{~s}^{-1}\right]$

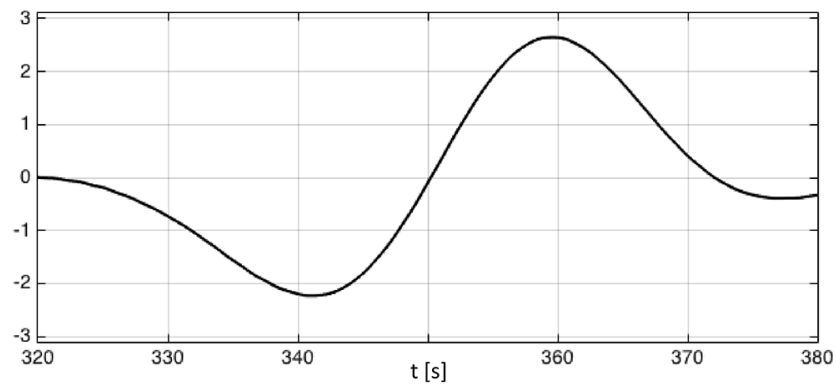

Figure 10. Illustration of finite-frequency travel-time shifts induced by a $38 \%$ density perturbation. Top row: Wave field snapshots before, during and after the wavefront interacts with the density heterogeneity, marked by the dashed box. The interaction with the density heterogeneity causes reflections and amplitude changes of the direct wave. The onset of the wavefront, however, remains unaffected. Bottom row: Synthetic seismograms taken at the position of the black circle in the top-row snapshots. The actual onset time of the waveforms at around $320 \mathrm{~s}$ is identical for simulations with (red) and without (black) density heterogeneity. However, the waveform differences, plotted to the right, induce a cross-correlation time shift of $\sim 1 \mathrm{~s}$. Locally, for example around $332 \mathrm{~s}$, the time shift reaches $1.4 \mathrm{~s}$.

for long-wavelength density heterogeneities especially indicates that the scattering is the dominant mechanism to produce these waveform differences. The energy transfer between the different components and towards later arrivals is also scattering-related.

As we show, the behaviour of density-induced misfits can be most often explained by an interplay of two physical parameters: the amount of scattering and the relative propagation distance. However, not all of the observed features can be interpreted on this ground. For instance, travel-time variations do not seem to exhibit a pronounced frequencydependence, in contrast to amplitude variations that decay rapidly with decreasing frequency (see Sect. 3.2 and Fig. 6. Since the travel times of seismic waves are exactly independent of density in the infinite-bandwidth approximation of ray theory (Cerveny, 2001), the travel-time differences observed in our experiments are most likely due to finite-frequency effects. Travel-time differences measured by cross-correlation within a finite frequency band are controlled by the complex interference of direct and scattered waves, which may lead to seemingly paradoxical effects (e.g. Tong et al., 1998; Marquering et al., 1999; Dahlen et al., 2000). This may include the large negative travel-time differences shown in Fig. 7.

Figure 10 illustrates finite-frequency travel-time changes for a 2-D $P$-SV wave field simulation. While interacting with a density anomaly, the wave field undergoes reflections and amplitude changes. The actual onset time of the wavefront remains largely unaffected. However, the waveform differences translate into a cross-correlation travel-time shift of $\sim 1 \mathrm{~s}$.

We should also take into account the possible signal processing artefacts that may play a role in our analysis. In comparing between different frequency bands, we are effectively changing the bandwidth used. Therefore, some of the large time shifts that are visible in the lower frequency histogram in Fig. 6 could be a result of the existence of non-zero crosscorrelation maxima for a narrow bandwidth, which would be purely signal-processing-related and have no physical interpretation. We do not expect to observe this effect in the broader frequency bands.

Naturally, scattering is a complex process that depends on the ratio of the scatterer size to the wavelength, the strength of scatterers and the source power (Groenenboom and Snieder, 1995). The signal processing artefacts and physical scattering signatures are not easily separated. Investigating whether the misfits accumulate later in the coda, which would indicate that they are scattering-related, and determining an optimal processing bandwidth size in order to decrease the amount of artificial large cross-correlation maxima, could be favourable. 
The density-induced waveform differences that we found in our numerical experiments are above the noise level of many of today's regional-scale seismic recordings. While this indicates that density heterogeneities do leave a measurable imprint, it does not automatically imply that crustal density structure can be easily recovered in a tomographic inversion. Trade-offs with $P$ and $S$ velocity structure, for instance, may prevent the unambiguous reconstruction of density heterogeneities. The resolvability of density structure may be analysed using principal component analysis of finite-frequency kernels (Sieminski et al., 2009), and it may be improved by the construction of targeted misfit functionals (Backus and Gilbert, 1968, 1970; Bernauer et al., 2014).

Finally, we note that the amplitude of the secondary wave field scattered off density heterogeneities may have similar or smaller amplitudes than globally propagating waves, e.g. PcP, PcS or ScS. Therefore, care needs to be taken when wave propagation is modelled regionally (e.g. Cupillard et al., 2012; Gokhberg and Fichtner, 2016).

\subsection{Random models of plausible Earth structure}

In the absence of detailed information on crustal density structure on regional scales, we base our numerical experiments on realisations of random Earth models. To ensure that the random models are plausible, we translate rms variations in $S$ velocity in the Anatolia model of Fichtner et al. (2013) into variations of $P$ velocity and density, using the empirical velocity-density scaling of Brocher (2005). The plausibility of the random models is limited by three factors: (i) the variability of regional-scale rms variations in $S$ velocity, (ii) the poorly known amplitude spectrum of velocity and density variations in the crust, and (iii) the range of different velocity-density scalings proposed in the literature. The rms variations in $S$ velocity in the Anatolian crust are $\sim 260 \mathrm{~m} \mathrm{~s}^{-1}$, with a horizontal correlation length of $\sim 200 \mathrm{~km}$ and a vertical correlation length of $\sim 20 \mathrm{~km}$. These correlation lengths were used for most of the numerical experiments, except for those in Sect. 3.4, where we studied the effect of medium complexity. Similar $S$ velocity variations on the order of $\pm 10 \%$ over similar distances were found in tomographic studies of other regions, including the Iberian Peninsula (e.g. El Moudnib et al., 2015; Fichtner and Villaseñor , 2015), California (e.g. Shapiro et al., 2005; Tape et al., 2010), the Caribbean plate (e.g. Gaite et al., 2015), or East Asia (e.g. Chen et al., 2015). This suggests that the rms variations of $S$ velocity variations with $200 \mathrm{~km}$ lateral and $20 \mathrm{~km}$ vertical correlation length are representative of real crustal structure at least in some regions.

For simplicity, we assume that the amplitude spectrum of the crustal velocity variations is white, meaning that velocity and density variations have nearly identical power at all scales considered in this study, i.e. from 50 to $1000 \mathrm{~km}$. The resulting velocity and density variations may be too large or too small by several percent, depending on whether a specific region is stable in the long term and subject to recent tectonic activity.

Uncertainties in velocity-density scalings are mostly caused by the natural scatter of the velocity-density relation in natural rocks. While $S$ velocity typically varies less than $\sim 10 \%$ for a given crustal $P$ velocity, density can easily vary by more than $50 \%$ for a given $v_{\mathrm{P}} / v_{\mathrm{S}}$ ratio (Brocher, 2005). Despite the natural scatter, published velocity-density relationships for the continental crust as a whole show good agreement with their respective range of validity (e.g. Ludwig et al., 1970; Gardner et al., 1974; Christensen and Mooney, 1995), suggesting that the choice of a particular one does not introduce a significant bias.

In the light of these uncertainties, it must be kept in mind that the waveform variations resulting from our synthetic random density heterogeneities represent a first rough estimate. It is intended to reveal the first-order effects but not the smaller details that certainly depend on the characteristics of a specific region.

\subsection{Velocity bias estimation}

The shifts in travel time observed here as a result of density structure may cause a bias in velocity structure obtained in tomographic models. In order to obtain an estimate of these velocity biases, we take a simplified approach. We consider the highest frequency band from 0.02 to $0.125 \mathrm{~Hz}$ and the reference medium with 200 lateral and $20 \mathrm{~km}$ vertical correlation length, previously used in Sect. 3.3 on the effect of epicentral distance. Since we do not analyse specific seismic phases, we take the time-shift variances for different epicentral distances as representative values of time shifts induced by 3-D density structure. Furthermore, we assume that sensitivity to velocity structure is concentrated on the great circle connecting source and receiver.

Based on these simplifications, we estimate that the shear velocity bias for an epicentral distance of $200 \mathrm{~km}$ is $25 \mathrm{~m} \mathrm{~s}^{-1}$ or $\sim 0.78 \%$ relative to the upper-crustal shear velocity of PREM (Dziewoński and Anderson, 1981). For epicentral distances of $1000 \mathrm{~km}$, the same bias is $\sim 21 \mathrm{~m} \mathrm{~s}^{-1}$ or $\sim 0.66 \%$. It follows that the velocity biases induced by the neglect of 3-D density variations are small compared to the crustal velocity variations inferred from travel-time tomography, which are on the order of $10 \%$. However, depending on the tomographic resolution and data quality, the biases may be larger than the error bars. A qualitative look at the detrimental effects that incorrect density information has on the wave speed models has recently been shown by Yuan et al. (2015).

\subsection{Attenuation bias estimation}

To quantify potential biases in attenuation induced by unknown 3-D density structure, we adopt similar simplifications as in the previous section. In the ray theory approxima- 


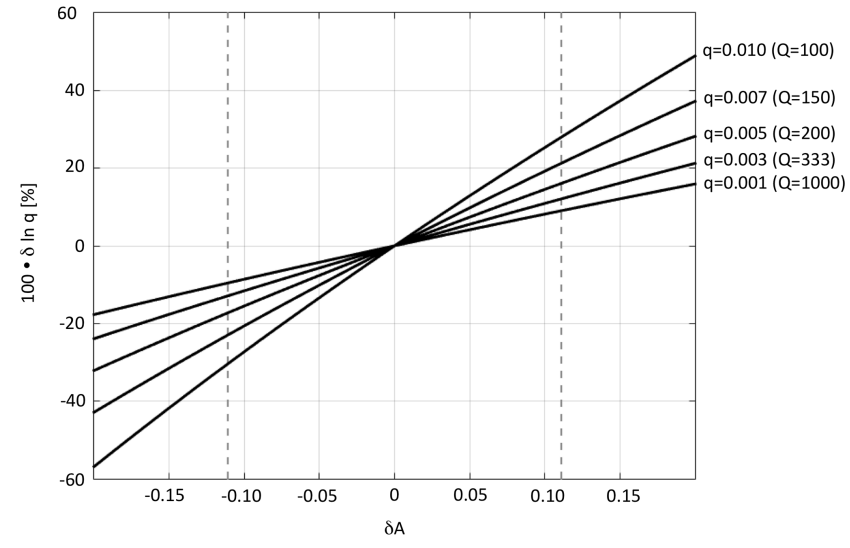

Figure 11. Fractional attenuation bias $\delta \ln q$ induced by 3-D density variations as a function of the relative amplitude difference $\delta A$. Dashed lines indicate the standard deviation of $\delta A$ for the setup that we used previously to study the effect of epicentral distance (epicentral distance: $1000 \mathrm{~km}$, frequency band: $0.02-0.125 \mathrm{~Hz}$, lateral correlation length: $200 \mathrm{~km}$, vertical correlation length: $20 \mathrm{~km}$; see Sect. 3.3). The corresponding histograms are shown in Fig. 8.

tion, relative amplitude differences between attenuated and attenuation-free waves are given by

$A=e^{\frac{-\pi r f q}{v}}$

with the background attenuation or inverse quality factor denoted by $q$, the epicentral distance by $r$, frequency by $f$, and velocity by $v$. The presence of 3-D density heterogeneity induces additional relative amplitude differences, $\delta A$, that translate into apparent variations in attenuation $\delta q$,

$A+\delta A=e^{\frac{-\pi r f(q+\delta q)}{v}}$.

Combining Eqs. (4) and (5) yields the fractional apparent attenuation bias $\delta \ln q$ as a function of $\delta A$,

$\delta \ln q=\left[\frac{\ln (A+\delta A+1)}{\ln (A+1)}-1\right]$.

Equation (6) reveals that attenuation biases for a given $\delta A$ have a dependence on the background attenuation $q$ through $A=e^{\frac{-\pi r f q}{v}}$. The bias is larger for larger attenuation. Figure 11 shows fractional attenuation biases $\delta \ln q$ as a function of the background attenuation $q$ for an epicentral distance of $1000 \mathrm{~km}$, the highest frequency band from 0.02 to $0.125 \mathrm{~Hz}$, and the medium with $200 \mathrm{~km}$ lateral and $20 \mathrm{~km}$ vertical correlation length. Amplitude variations for this setup are summarised in the right panel of Fig. 8.

Taking the variance of the relative amplitude differences of 0.11 as a representative value (see Sect. 3.3), the apparent variations in attenuation, $\delta \ln q$, approximately range between \pm 20 and $\pm 60 \%$ for $q$ between 0.001 and 0.010 ( $Q$ between 100 and 1000). It follows that the apparent variations in attenuation induced by 3-D density structure can be on the order of tens of percent, thus being comparable to attenuation heterogeneities found on regional and global scales (e.g. Mitchell, 1995; Romanowicz, 1995; Haberland and Rietbrock, 2001; Selby and Woodhouse, 2002; Dalton et al., 2008; Kennett and Abdullah, 2011; Trampert and Fichtner, 2013; Zhu et al., 2015). This result highlights that 3-D density structure should be taken into account when using fullwaveform techniques to invert for 3-D variations in attenuation.

\section{Conclusions}

We presented a series of numerical experiments to study the effect of 3-D crustal density heterogeneities on regional seismic wave propagation in the frequency range from 0.02 to $0.125 \mathrm{~Hz}(8-50 \mathrm{~s})$. These were intended to (i) reveal the extent to which density-induced waveform perturbation may be measurable, and (ii) facilitate a better intuitive understanding of the underlying wave propagation physics.

While numerical experiments can of course never be exhaustive, our series of tests still allows us to make a limited number of general statements: for media with $200 \mathrm{~km}$ lateral correlation length, travel-time perturbations can exceed $\sim 1 \mathrm{~s}$ over an epicentral distance of $\sim 1000 \mathrm{~km}$. Amplitude perturbations for the same scenario can be several tens of percent. With decreasing frequency, amplitude perturbations decrease rapidly, but travel-time perturbations remain at approximately the same level. This indicates that the observed travel-time variations are a finite-frequency effect, i.e. a local change of amplitudes that manifests itself as a time shift, when the time shift is measured by cross-correlation.

Both amplitude and travel-time variations increase with increasing epicentral distance. This indicates that density does not only have a local effect, which is an essential prerequisite for the applicability of tomographic methods to constrain 3 -D density in the crust. Waveform perturbations clearly increase with increasing medium complexity. They are practically negligible in transmission mode, i.e. when the correlation length of the medium heterogeneities is much larger than the wavelength. However, when the correlation length approaches the wavelength, density-induced waveform perturbations can be observed easily. Recent regional-scale fullwaveform inversions operate in a regime where resolved heterogeneities have characteristic sizes comparable to the wavelength (e.g. Tape et al., 2010; Fichtner et al., 2013).

Our most important finding is that waveform perturbations induced by realistic crustal density variations can certainly be observed in modern, high-quality regional seismic data. While travel-time differences of typically less than $1 \mathrm{~s}$ will often be small compared to travel-time differences caused by velocity heterogeneities, amplitude variations of more than $10 \%$ are comparable with those induced by $3-\mathrm{D}$ velocity structure and attenuation. This implies, on the one hand, that density structure may to some extent be constrained in fu- 
ture full-waveform inversions. On the other hand it suggests that current full-waveform inversions that use amplitude information may be biased due to the neglect of 3-D variations in density.

\section{Data availability}

The wave propagation package SES3D is a free open source software released under Apache 2.0 License. It is available for download at: http://www.cos.ethz.ch/software/ses3d. html.

The synthetic data used in this study, along with python tools for random media generation, signal comparison and histogram stacking, are to be found at: doi:10.5281/zenodo.168576, (Płonka, 2016). 


\section{Appendix A: Random model generation}

We generate random media with the Fourier method, widely used in seismological research (e.g. Frankel and Clayton, 1986; Frankel, 1989; Igel and Gudmundsson, 1997; Klimeš, 2002; Jahnke et al., 2008), and recently extended to nonstationary and anisotropic media by Meschede and Romanowicz (2015). For this, we generate a random, uniformly distributed phase spectrum $\varphi(\mathbf{k})$ between $-\pi$ and $\pi$, with $\mathbf{k}$ being the 3-D wavenumber. The random spectrum $e^{i \varphi(\mathbf{k})}$ is then modulated by a positive, real-valued filter $f(\mathbf{k})$ to yield the wavenumber-domain random model

$m(\mathbf{k})=f(\mathbf{k}) e^{i \varphi(\mathbf{k})}$.

The filter $f(\mathbf{k})$ is designed such that it is flat below $k_{\max }$ and wavenumber components $k_{i}$ above a given threshold $k_{i, \max }=$ $2 \pi / \lambda_{i, \min }$ are excluded. Computing the inverse Fourier transform yields the space-domain random model

$m(\mathbf{x})=\frac{1}{(2 \pi)^{3}} \iiint m(\mathbf{k}) e^{i \mathbf{k} \cdot \mathbf{x}} d \mathbf{k}$.

By design, the 3-D field $m(\mathbf{x})$ only contains wavelengths above $\lambda_{i, \min }$ in the $i$-direction. Finally, the random realisation $m(\mathbf{x})$ is appropriately scaled and assigned to a specific medium parameter, such as density, $P$ - or $S$-velocity. 
Author contributions. Agnieszka Płonka performed all 3-D numerical wave propagation experiments. All three authors were involved in the design of the experiments, the interpretation of results and the manuscript writing.

Acknowledgements. The authors would like to thank Hanneke Paulssen, Ivan Pires de Vasconcelos and Jeannot Trampert for numerous interesting discussions, and two anonymous reviewers for their constructive comments. This research was supported by the Swiss National Supercomputing Center (CSCS) in the form of the GeoScale and $\mathrm{CH} 1$ projects, and by the Netherlands Organisation for Scientific Research (VIDI grant 864.11.008).

Edited by: M. Malinowski

Reviewed by: two anonymous referees

\section{References}

Aki, K. and Richards, P.: Quantitative Seismology, University Science Books, 2002.

Anderson, D. L., Schreiber, E., Lieberman, R. C., and Soga, N.: Some elastic constant data on minerals relevant to geophysics, Rev. Geophys. Space Phys., 6, 491-524, 1968.

Backus, G. E. and Gilbert, F.: The resolving power of gross Earth data, Geophys, J. Roy. Astr. Soc., 16, 169-205, 1968.

Backus, G. E. and Gilbert, F.: Uniqueness in the inversion of inaccurate gross Earth data, Philos. T. R. Soc. A, 266, 123-192, 1970.

Becker, T. W. and Boschi, L.: A comparison of tomographic and geodynamic mantle models, Geochem. Geophys. Geosys., 3, 1525-2027, doi:10.1029/2001GC000168, 2002.

Bernauer, M., Fichtner, A., and Igel, H.: Optimal observables for multi-parameter seismic tomography, Geophys. J. Int., 198, 1241-1254, 2014.

Bozdağ, E., Trampert, J., and Tromp, J.: Misfit functions for full waveform inversion based on instantaneous phase and envelope measurements, Geophys. J. Int., 185, 845-870, 2011.

Brocher, T. M.: Empirical relations between elastic wavespeeds and density in the Earth's crust, Bull. Seis. Soc. Am., 95, 2081-2092, 2005.

Bunge, H.-P., Hagelberg, C. R., and Travis, B. J.: Mantle circulation models with variational data assimilation: Inferring past mantle flow and structure from plate motion histories and seismic tomography, Geophys. J. Int., 152, 280-301, 2003.

Cerveny, V.: Seismic ray theory, Cambridge University Press, 2001.

Chen, P., Zhao, L., and Jordan, T. H.: Full 3-D tomography for the crustal structure of the Los Angeles region, Bull. Seismol. Soc. Am., 97, 1094-1120, 2007.

Chen, M., Niu, F., Liu, Q., Tromp, J., and Zheng, X.: Multiparameter adjoint tomography of the crust and upper mantle beneath East Asia - Part I: Model construction and comparisons, J. Geophys. Res., 120, 1762-1786, 2015.

Christensen, N. I. and Mooney, W. D.: Seismic velocity structure and composition of the continental crust: a global view, J. Geophys. Res., 1000, 9761-9788, 1995.

Cupillard, P., Delavaud, E., Burgos, G., Festa, G., Vilotte, J.-P., Capdeville, Y., and Montagner, J.-P.: RegSEM: A versatile code based on the spectral element method to compute seismic wave propagation at the regional scale, Geophys. J. Int., 188, 12031220, 2012.

Dahlen, F., Hung, S.-H., and Nolet, G.: Fréchet kernels for finitefrequency traveltimes - I. Theory, Geophys. J. Int., 141, 157174, 2000.

Dahlen, F. A. and Tromp, J.: Theoretical Global Seismology, Princeton University Press, NJ, 1998.

Dalton, C. A., Ekström, G., and Dziewonski, A. M.: The global attenuation structure of the upper mantle, J. Geophys. Res., 113, B09303, doi:10.1029/2007JB005429, 2008.

Dziewoński, A. M., Hager, B. H., and O’Connell, R. J: Large-scale heterogeneities in the lower mantle, J. Geophys. Res., 82, 239255, 1977.

Dziewoński, A. M. and Anderson, D. L.: Preliminary reference Earth model, Phys. Earth Planet. Int., 25, 297-356, 1981.

El Moudnib, L., Villaseñor, A., Harnafi, M., Gallart, J., Pazos, A., Serrano, I., nd J. A. Pulgar, D. C., Ibarra, P., Himmi, M. M., and Chourak, M.: Crustal structure of the Betic-Rif system, western Mediterranean, from local earthquake tomography, Tectonophysics, 643, 94-105, 2015.

Faccioli, E., Maggio, F., Quarteroni, A., and Tagliani, A.: Spectraldomain decomposition methods for the solution of acoustic and elastic wave equations, Geophysics, 61, 1160-1174, 1996.

Fichtner, A., Kennett, B. L. N., Igel, H., and Bunge, H.-P.: Theoretical background for continental- and global-scale full-waveform inversion in the time-frequency domain, Geophys. J. Int., 175, 665-685, 2008.

Fichtner, A., Kennett, B. L. N., Igel, H., and Bunge, H.-P.: Full seismic waveform tomography for upper-mantle structure in the Australasian region using adjoint methods, Geophys. J. Int. , 179, 1703-1725, 2009.

Fichtner, A., Kennett, B. L. N., Igel, H., and Bunge, H.-P.: Spectralelement simulation and inversion of seismic waves in a spherical section of the Earth, J. Num. An. Ind. Appl. Math., 4, 11-22, 2009.

Fichtner, A.: Full Seismic Waveform Modelling and Inversion, Springer, Heidelberg, 2010.

Fichtner, A., Saygin, E., Taymaz, T., Cupillard, P., Capdeville, Y., and Trampert, J.: The deep structure of the North Anatolian Fault Zone, Earth Planet. Sc. Lett., 373, 109-117, 2013.

Fichtner, A. and Villaseñor, A.: Crust and upper mantle of the western Mediterranean - Constraints from full-waveform inversion, Earth Planet. Sc. Lett., 428, 52-62, 2015.

Fichtner, A., Trampert, J., Cupillard, P., Saygin, E., Taymaz, T., Capdeville, Y.. and Villaseñor, A.: Multi-scale full waveform inversion, Geophys. J. Int., 194, 534-556, 2013.

Frankel, A.: A review of numerical experiments on seismic wave scattering, Pure Appl. Geophys., 4, 639-685, 1989.

Frankel, A. and Clayton, R.: Finite-difference simulations of seismic scattering: implications for the propagation of short period seismic waves in the crust and models of crustal heterogeneity, J. Geophys. Res., 91, 6465-6489, 1986.

Furumura, T. and Kennett, B. L. N.: Subduction zone guided waves and the heterogeneity structure of the subducted plate - intensity anomalies in northern Japan, J. Geophys. Res., 110, B10302, doi:10.1029/2004JB003486, 2005.

Gaite, B., Villaseñor, A., Iglesias, A., Herraiz, M. and JiménezMunt, I.: A 3-D shear velocity model of the southern North 
American and Caribbean plates from ambient noise and earthquake tomography, Solid Earth, 6, 271-284, 2015.

Gardner, G. H. F., Gardner, L. W., and Gregory, A. R.: Formation velocity and density - the diagnostic basics for stratigraphic traps, Geophysics, 39, 770-780, 1974.

Gokhberg, A. and Fichtner, A.: Full-waveform inversion on heterogeneous HPC systems, Comput. Geosci., 89, 260-268, doi:10.1016/j.cageo.2015.12.013, 2016.

Groenenboom, J. and Snieder, R.: Attenuation, dispersion and anisotropy by multiple scattering of transmitted waves through distributions of scatterers, J. Acoust. Soc. Am., 98, 3482-3492, 1995.

Haberland, C. and Rietbrock, A.: Attenuation tomography in the western central Andes: A detailed insight into the structure of a magmatic arc, J. Geophys. Res., 106, 11151-11167, 2001.

Igel, H. and Gudmundsson, O.: Frequency-dependent effects on travel times and waveforms of long-period S and SS waves, Phys. Earth. Planet. Int., 104, 229-246, 1997.

Ishii, M. and Tromp, J.: Normal-mode and free-air gravity constraints on lateral variations in velocity and density of Earth's mantle, Science, 285, 1231-1236, 1999.

Ishii, M. and Tromp, J.: Even-degree lateral variations in the Earth's mantle constrained by free oscillations and the free-air gravity anomaly, Geophys. J. Int., 145, 77-96, 2001.

Ishii, M. and Tromp, J.: Constraining large-scale mantle heterogeneity using mantle and inner-core sensitive normal modes, Phys. Earth Planet. Inter., 146, 113-124, 2004.

Jahnke, G., Thorne, M. S., Cochard, A., and Igel, H.: Global SHwave propagation using a parallel axisymmetric spherical finitedifference scheme: application to whole-mantle scattering, Geophys. J. Int., 173, 815-826, 2008.

Jordan, T. H.: The continental tectosphere, Rev. Geophys., 13, 1-12, 1975.

Jordan, T. H.: Composition and development of the continental tectosphere, Nature, 274, 544-548, 1978.

Kennett, B. L. N.: The seismic wavefield I. - Introduction and theoretical development, Cambridge University Press, 2001.

Kennett, B. L. N. and Furumura, T.: Stochastic waveguide in the lithosphere: Indonesian subduction zone to Australian craton, Geophys. J. Int., 172, 363-382, 2008.

Kennett, B. L. N. and Bunge, H.-P.: Geophysical Continua, Cambridge University Press, 2008.

Kennett, B. L. N. and Abdullah, A.: Seismic wave attenuation beneath the Australasian region, Austr. J. Earth Sci., 58, 285-295, 2011.

Klimeš, L.: Correlation functions of random media, Pure Appl. Geophys., 159, 1811-1831, 2002.

Koelemeijer, P., Deuss, A. , and Ritsema, J.: Density structure of Earth's lowermost mantle from stoneley mode splitting observations, Nat. Commun., under review, 2016.

Komatitsch, D. and Tromp, J.: Introduction to the spectral element method for three-dimensional seismic wave propagation, Geophys. J. Int., 139, 806-822, 1999.

Komatitsch, D. and Vilotte, J. P.: The spectral element method: an effective tool to simulate the seismic response of 2-D and 3-D geological structures, Bull. Seismol. Soc. Am, 88, 368-392, 1998.

Kristekova, M., Kristek, J., and Moczo, P.: Time-frequency misfit and goodness-of-fit criteria for quantitative comparison of time signals, Geophys. J. Int., 178, 813-825, 2009.
Kristekova, M., Kristek, J., Moczo, P., and Day, S. M.: Misfit criteria for quantitative comparison of seismograms, Bull. Seismol. Soc. Am., 96, 1836-1850, 2006.

Kuo, C. and Romanowicz, B.: On the resolution of density anomalies in the Earth's mantle using spectral fitting of normal mode data, Geophys. J. Int., 150, 162-179, 2002.

Lin, F.-C. and Tsai, B. S. V. C.: Joint inversion of Rayleigh wave phase velocity and ellipticity using USArray: Constraining velocity and density structure in the upper crust, Geophy Res. Lett., 39, L12303, doi:10.1029/2012GL052196, 2012.

Lin, F.-C., Tsai, V. C., and Ritzwoller, M. H.: The local amplification of surface waves: A new observable to constrain elastic velocities, density, and anelastic attenuation, J. Geophys. Res., 117, B06302, doi:10.1029/2012JB009208, 2012.

Liu, L. and Gurnis, M.: Simultaneous inversion of mantle properties and initial conditions using an adjoint of mantle convection, J. Geophys. Res., 113, B08405, doi:10.1029/2008JB005594, 2008.

Ludwig, W. J., Nafe, J. E., and Drake, C. L.: Seismic refraction, edited by: Maxwell, A. E., The Sea, Wiley-Interscience, New York, 1970.

Maceira, M. and Ammon, C. J.: Joint inversion of surface wave velocity and gravity observations and its application to central asian basins shear velocity structure, J. Geophys. Res., 114, B02314, doi:10.1029/2007JB005157, 2009.

Marquering, H., Dahlen, F. A. , and Nolet, G.: Three-dimensional sensitivity kernels for finite-frequency traveltimes: the bananadoughnut paradox, Geophys. J. Int., 137, 805-815, 1999.

Meschede, M. and Romanowicz, B.: Non-stationary spherical random media and their effect on long-period mantle waves, Geophys. J. Int., 203, 1605-1625, 2015.

Mitchell, J. B.: Anelastic structure and evolution of the continental crust and upper mantle from seismic surface wave attenuation, Rev. Geophys., 33, 441-462, 1995.

Mosca, I., Cobden, L., Deuss, A., Ritsema, J., and Trampert, J.: Seismic and mineralogical structures of the lower mantle from probabilistic tomography, J. Geophys. Res., 117, B06304, doi:10.1029/2011JB008851, 2012.

Nataf, H. C. and Ricard, Y.: 3SMAC: an a priori tomographic model of the upper mantle based on geophysical modelling, Phys. Earth Planet. Int., 95, 101-122, 1996.

Peter, D., Komatitsch, D., Luo, Y., Martin, R., Le Goff, N., Casarotti, E., Le Loher, P., Magnoni, F., Liu, Q., Blitz, C., Nissen-Meyer, T., Basini, P., and Tromp, J.: Forward and adjoint simulations of seismic wave propagation on fully unstructured hexahedral meshes, Geophys. J. Int., 186, 721-739, 2011.

Płonka, A.: The imprint of crustal density heterogeneities on regional seismic wave propagation - dataset, doi:10.5281/zenodo.168576, 2016.

Resovsky, J. and Ritzwoller, M.: Regularisation uncertainty in density models estimated from normal mode data, Geophys. Res. Lett., 26, 2319-2322, 1999.

Resovsky, J. and Trampert, J.: Reliable mantle density error bars: an application of the Neighbourhood Algorithm to normal-mode and surface wave data, Geophys. J. Int., 150, 665-672, 2002.

Richards, M. and Lithgow-Bertelloni, C.: A geodynamic model of mantle density heterogeneity, J. Geophys. Res., 98, 21-895, 1993. 
Rickers, F., Fichtner, A., and Trampert, J.: Imaging mantle plumes with instantaneous phase measurements of diffracted waves, Geophys. J. Int., 19, 650-664, 2012.

Rickers, F., Fichtner, A., and Trampert, J.: The Iceland - Jan Mayen plume system and its impact on mantle dynamics in the North Atlantic region: Evidence from full-waveform inversion, Earth Planet. Sc. Lett., 367, 39-51, 2013.

Romanowicz, B.: A global tomographic model of shear attenuation in the upper mantle, J. Geophys. Res., 100, 12375-12394, 1995.

Romanowicz, B.: Can we resolve 3-D density heterogeneity in the lower mantle?, Geophys. Res. Lett., 28, 1107-1110, 2001.

Romanowicz, B. and Mitchell, B. J.: Deep Earth structure $-Q$ of the Earth from Crust to Core, edited by: Schubert, G., Treatise on Geophysics, 731-774, Elsevier 2007.

Selby, N. D. and Woodhouse, J. H.: The Q structure of the upper mantle: Constraints from Rayleigh wave amplitudes, J. Geophys. Res., 107, ESE 5-1-ESE 5-11, doi:10.1029/2001JB000257, 2002.

Shapiro, N. M., Campillo, M., Stehly, L., and Ritzwoller, M.: High resolution surface wave tomography from ambient seismic noise, Science, 307, 1615-1618, 2005.

Sieminski, A., Trampert, J., and Tromp, J.: Principal component analysis of anisotropic finite-frequency kernels, Geophys. J. Int., 179, 1186-1198, 2009.

Simmons, N. A., Forte, A. M., Boschi, L., and Grand, S. P.: GyPSuM: A joint tomography model of mantle density and seismic wave speeds, J. Geophys. Res., 115, B12310, doi:10.1029/2010JB007631, 2001.

Tanimoto, T.: Waveform inversion for three-dimensional density and S wave structure, J. Geophys. Res., 96, 8167-8189, 1991.

Takeuchi, H. and Saito, M.: Seismic surface waves, in: Methods in Computational Physics, edited by: Bolt, B. A., 11, 217-295, 1972.

Tape, C., Liu, Q., Maggi, A., and Tromp, J.: Seismic tomography of the southern California crust based upon spectral-element and adjoint methods, Geophys. J. Int., 180, 433-462, 2010.

Tarantola, A.: A strategy for nonlinear elastic inversion of seismic reflection data, Geophysics, 51, 1893-1903, 1986.

Tondi, R., Achauer, U., Landes, M., Davi, R., and Besutiu, L.: Unveiling seismic and density structure beneath the Vrancea seismogenic zone, Romania, J. Geophys. Res., 141, B11307, doi:10.1029/2008JB005992, 2009.
Tondi, R., de Franco, R., and Barzaghi, R.: Sequential inversion of refraction and wide-angle reflection traveltimes and gravity data for two-dimensional velocity structures, Geophys. J. Int., 141, 679-698, 2000.

Tong, J., Dahlen, F. A., Nolet, G., and Marquering, H.: Diffraction effects upon finite-frequency travel times: A simple 2-D example, Geophys. Res. Lett., 25, 1983-1986, 1998.

Trampert, J., Deschamps, F., Resovsky, J. , and Yuen, D.: Probabilistic tomography maps chemical heterogeneities throughout the lower mantle, Science, 306, 853-856, 2004.

Trampert, J. and Fichtner, A.: Global imaging of the Earth's deep interior: seismic constraints on (an)isotropy, density and attenuation, edited by: Karato, S., Physics and Chemistry of the deep Earth, Wiley-Blackwell, 2013.

Tromp, J., Tape, C., and Liu, Q.: Seismic tomography, adjoint methods, time reversal and banana-doughnut kernels, Geophys. J. Int., 160, 195-216, 2005.

Turcotte, D. L. and Schubert, G.: Geodynamics, Cambridge University Press, 2014.

van Leeuwen, T. and Mulder, W. A.: A correlation-based misfit criterion for wave-equation traveltime tomography, Geophys. J. Int., 182, 1383-1394, 2010.

Warners-Ruckstuhl, K. N., Govers, R., and Wortel, R.: Lithospheremantle coupling and the dynamics of the Eurasian Plate, Geophys. J. Int., 189, 1253-1276, 2012.

Woodhouse, J. H.: The calculation of eigenfrequencies and eigenfunctions of the free oscillations of the Earth and the Sun, edited by: Doornbos, D. J., Seismological algorithms, Academic Press, New York, 321-370, 1988.

Woodhouse, J. H. and Deuss, A. F.: Theory and ObservationsEarth's Free Oscillations, in: Treatise on Geophysics, doi:10.1016/B978-044452748-6.00002-X, Elsevier 2007.

$\mathrm{Wu}, \mathrm{R}$. and Aki, K.: Scattering characteristics of elastic waves by an elastic heterogeneity, Geophysics, 50, 582-595, 1985.

Yuan, Y. O., Simons, F. J., and Bozdag, E.: Multiscale adjoint tomography for surface and body waves, Geophysics, 80, R281R302, 2015.

Zhu, H., Bozdağ, E., and Tromp, J.: Seismic structure of the European upper mantle based on adjoint tomography, Geophys. J. Int., 201, 18-52, 2015. 